\title{
Análise da Relação entre Quantidades e Custos Totais de Produção de Frango no Estado do Ceará
}

\author{
Jucimar Casimiro de Andrade \\ Mestrado em Administração pela Universidade Federal Rural de Pernambuco - UFRPE \\ Professor do Grupo Ser Educacional - SER \\ Rua Guilherme Pinto 114. Graças. Recife/PE. CEP: 52011-210 \\ E-mail: jucimarcandrade@gmail.com \\ Fernando Salvino da Silva \\ Especialização em Planejamento Organizacional pela Faculdade de Administração de \\ Pernambuco - FACAP \\ Professor do Grupo Ser Educacional - SER \\ Rua Guilherme Pinto 114. Graças. Recife/PE. CEP: 52011-210 \\ E-mail:administracao.gestao@sereducacional.com \\ Marcela Rebecca Pereira \\ Doutorado em andamento em Administração pela Universidade Federal do \\ Pernambuco - UFPE \\ Professora do Grupo Ser Educacional - SER \\ Rua Guilherme Pinto 114. Graças. Recife/PE. CEP: 52011-210 \\ E-mail: marcela.pereira@sereducacional.com \\ Robson José Silva Santana \\ Especialização em Contabilidade de Controladoria pela Universidade Federal de \\ Pernambuco - UFPE \\ Professor do Grupo Ser Educacional - SER \\ Rua Guilherme Pinto 114. Graças. Recife/PE. CEP: 52011-210 \\ E-mail:prof.robjss@gmail.com
}

\section{RESUMO}

Diante do atual cenário econômico que ora vivenciam os mercados mundiais e considerando a necessidade empresarial de mensurar e controlar melhor seus custos produtivos, principalmente no segmento de agronegócio da carne de frango de corte, o presente artigo teve como propósito analisar como os custos de produção de frango no estado do Ceará relacionam-se com as quantidades produzidas em unidades/lotes de frango para abate. Para isso, utilizaram-se os dados mensais dos custos de produção de frango disponíveis no site da Central de Inteligência de Aves e Suínos (CIAS), referentes ao estado do Ceará, entre os meses de janeiro de 2010 a setembro de 2015. Para os cálculos, utilizou-se uma adaptação do modelo desenvolvido por Carmo, Lima, Oliveira e Oliveira (2011) e as planilhas do Microsoft Exce/® , em conjunto com o pacote econométrico Gret/@ (Gnu Regression, Econometrics and Time-series Library). Portanto, no que se refere ao modelo estimado, em que a variável y1 representa os custos totais de produção (fixos e variáveis) e x1 representa as quantidades produzidas, 
Análise da Relação entre Quantidades e Custos Totais de Produção de Frango no Estado do Ceará Jucimar Casimiro de Andrade, Fernando Salvino da Silva, Marcela Rebecca Pereira, Robson

constatou-se que o modelo apresentou um erro de estimação em torno de $-6,59 \%$ para os dados de 2014; e, em relação às simulações nas planilhas feitas por meio da Técnica de Monte Carlo, observou-se que o modelo é satisfatório e serve como apoio à tomada de decisão sobre as projeções de custos/lotes para uma estimativa de produção de frangos para abate entre 1.000 e 5.000 cabeças, desde que se assuma uma margem de erro de em torno de $\pm 1,11 \%$.

Palavras-chave: Custos de produção de frango. Gestão do agronegócio. Modelagem matemática.

\section{Analysis about the Relationship Between Quantities and Total Costs of Chicken Production in Ceará State}

\section{ABSTRACT}

Owing to the current economic scenario in the world markets, and considering the business need to better measure and control its production costs, specially in the agribusiness segment of poultry meat, the purpose of this article was to analyze how the production costs of chicken in the state of Ceará are related to the quantities produced in units/lots of chicken for slaughter. For this, it has been used monthly data of chicken production costs, available on the website of Central de Inteligência de Aves e Suínos (CIAS), concerning to the state of Ceará in Brazil between the months of January 2010 to September 2015. For the estimations, it was made an adaptation of a model developed by the author Carmo, Lima, Oliveira e Oliveira (2011) and also was used the Microsoft Excel® spreadsheets, together with a statistical package called Econometrics and Time-series Library (Gretl). Therefore, in relation to the model, where the variable $\mathrm{y}_{1}$ represents the total production costs (fixed and variable costs) and $x_{1}$ represents the quantities produced, it has been verified that the model presented an estimation error around $-6.59 \%$ for the data of 2014 . According to the simulations in the spreadsheets made through the Monte Carlo technique, it has been observed that the model is satisfactory and serves as support for decision making on cost projections for an estimated production of chickens between 1.000 and 5.000 animals, assuming an error's margin around $\pm 1,11 \%$.

Keywords: Chicken production costs. Management of agribusiness. Mathematical modeling. 


\section{INTRODUÇÃO}

A produção pecuária do Brasil vem se destacando no cenário mundial. Vários fatores podem explicar sucessivos ganhos de produção da agropecuária brasileira, especialmente por se configurarem como elementos constitutivos da base econômica primária e que repercutem nos setores secundário e terciário, auxiliando no superávit da balança do agronegócio.

Oliveira (2009) destaca que o agronegócio brasileiro é uma atividade próspera, segura e rentável. Com um clima diversificado, chuvas regulares, energia solar abundante e quase $13 \%$ de toda a água doce disponível no planeta, o Brasil tem 388 milhões de hectares de terras agricultáveis férteis e de alta produtividade, dos quais 90 milhões ainda não foram explorados.

Como parte integrante do setor primário do agronegócio brasileiro, a produção de frango destaca-se como uma das molas propulsoras da produção pecuária juntamente com outras atividades que também se destacam no cenário nacional. Arruda e Santos (2017) destacam que as exportações brasileiras de carne de frango (considerando frango inteiro, cortes, processados e salgados) totalizaram, em 2015, uma produção de 13,146 milhões de toneladas, um volume 3,58\% superior à produção em 2014 . Esses dados indicam também que, mesmo com a crise econômica brasileira, o consumo per capita interno aumentou para uma média de 43,25 Kg/ano em 2015, 1,1\% maior que em 2014.

Assim, a produção de frango de corte, que antes era concentrada nas regiões Sul e Sudeste do país, tem migrado para outras regiões do país, nos últimos anos, principalmente para as proximidades das zonas produtoras de grãos e também para áreas mais próximas a centros consumidores de carne de frango, como as regiões metropolitanas da Bahia, de Pernambuco e do Ceará.

O estado do Ceará apresenta uma significativa potencialidade de crescimento do setor avícola, sobretudo pela proximidade de centros consumidores, como nos estados vizinhos Piauí, Paraíba e Rio Grande do Norte, e também por Fortaleza ser a capital 
mais próxima da Europa, o que facilita a produção e exportação de carne de frango para esse continente e para o oriente médio, hoje um dos principais consumidores de frango no mundo.

Santos, Marion e Segatti (2009) afirmam que a importância da atividade avícola no estado do Ceará pode ser dimensionada pelos números gerados: faturamento de cerca de $\mathrm{R} \$ 700$ milhões em 2015; representatividade do valor bruto da atividade avícola de aproximadamente $2,5 \%$ do Produto Interno Bruto (PIB) estadual.

O conhecimento sobre os custos de produção agrícola é uma importante ferramenta de controle e gerenciamento das atividades produtivas, de geração de informações para subsidiar a tomada de decisão pelos produtores rurais e, também, como auxílio à formulação de estratégias pelo setor público para esse segmento econômico.

Assim, para administrar com eficiência e eficácia uma unidade produtiva agrícola, é imprescindível, entre outras variáveis, o domínio da tecnologia e do conhecimento dos gastos com insumos e serviços em cada fase produtiva da lavoura, que tem no custo um indicador importante das escolhas do produtor (Santos et al., 2009).

Destarte, a presente investigação se justifica, pois o Brasil é um dos maiores produtores de carne de frango de corte que serve para abastecer o mercado interno e externo e que representa um importante item na formação da balança comercial. Portanto, estudos que venham a melhorar a viabilidade econômica desse segmento são importantes, pois proporcionam ganho de produção sem necessariamente representar aumento dos custos de produção incorridos.

Justifica-se ainda, pois o estado do Ceará é um dos maiores produtores de carne de frango do Nordeste, e estudos que venham a aumentar a eficiência na produção e comercialização de frango naquele estado podem representar um diferencial competitivo e auxiliar na melhoria do processo produtivo tanto para o produtor integrado quanto para a agroindústria.

O estudo está estruturado da seguinte forma: introdução, com uma abordagem panorâmica geral da importância do agronegócio brasileiro; o referencial teórico está 
dividido em três seções que tratam sobre conceitos usuais em custos e suas várias conotações, além de estudos e trabalhos correlatos ao problema aqui levantado; finalizando, o trabalho com o método escolhido para resolução da questão-problema e com as considerações finais sobre os achados das análises feitas.

Ante os argumentos expostos, este estudo visa responder à seguinte questãoproblema: como os custos totais de produção de frango (fixos e variáveis) no estado do Ceará relacionam-se com as quantidades produzidas em unidades/lotes de frango para abate?

\section{REFERENCIAL TEÓRICO}

Este tópico do trabalho versa sobre a gestão de custos da atividade pecuária especificamente na produção de frango de corte no Brasil e no estado do Ceará, apontando alguns trabalhos empíricos já realizados e as discussões atualmente levantas na literatura.

\subsection{Gestão de Custos}

Compreender o comportamento dos custos de produção em uma determinada atividade pode envolver alguns desafios, principalmente se o proprietário não tiver conhecimento técnico sobre o assunto, o que pode elevar os riscos de um insucesso na atividade. Assim, a compreensão de como os custos tendem a se comportar ao longo de uma série histórica conhecida pode auxiliar o produtor na hora da tomada de decisão e também ajudar a avaliar melhor o efeito de políticas internas que a empresa adota.

Gestão de custos é o ramo da contabilidade que define questões de custos estratégicos que são movidos tanto por informações financeiras quanto não financeiras. O objetivo, portanto, da gestão de custos é fornecer condições para as empresas tomarem decisões, a fim de aumentarem a competitividade da empresa (Martins, 2003)

Reis (2002) define os custos de produção como sendo a soma dos valores de todos os recursos (insumos e serviços) utilizados no processo produtivo de uma 
atividade agrícola, em certo período de tempo e que podem ser classificados como de curto e de longo prazo.

A estimativa dos custos de produção é o detalhamento de todas as despesas e receitas diretas e indiretas das atividades produtivas (Garcia, 2005). Esse custo é um dos principais fatores a ser analisado para a boa administração de uma empresa. Com ele, pode-se estimar a viabilidade econômica de um negócio, em função do capital investido, compará-lo com outras atividades agropecuárias, ou mesmo, com atividades que não envolvam uso da terra.

Sobre a importância da gestão eficiente e eficaz dos custos de produção, Ferreira (2004) destaca:

A importância de estudar o custo de produção deve-se a dois objetivos principais: auxiliar no processo de tomada de decisão do produtor e avaliar os efeitos das políticas governamentais. Para o produtor, o conhecimento detalhado dos componentes do custo auxilia a determinação do sistema de produção a ser usado, relacionado com a utilização dos diversos fatores e levando em consideração a sua disponibilidade e preço. Assim, o produtor poderá alterar sua planilha de custo, racionalizando a utilização de fatores e objetivando a maximização do lucro. Para o governo, a intervenção dar-se-á mediante políticas que objetivem o controle de preço e de crédito, entre outras.

Sem conhecer os custos, o empresário não saberá se está efetivando ou não os lucros, nem terá subsídios para tomar decisões corretas com o intuito de direcionar sua empresa aos resultados positivos ou melhores que os atuais (Nogueira, 2004).

O comportamento dos custos caracteriza a forma como os mesmos se modificam a partir de mudanças nos níveis de atividade ou no volume de produção, ou seja, o custo muda quando uma atividade muda (Gomes, De Lima, \& Steppan, 2007). Para esse comportamento, é feita a distinção entre custos fixos e custos variáveis dentro de um processo produtivo. Obviamente, os custos variáveis variam quando a quantidade aumenta e custos fixos se mantêm estáveis, independentemente de quanto se produz. 
Análise da Relação entre Quantidades e Custos Totais de Produção de Frango no Estado do Ceará Jucimar Casimiro de Andrade, Fernando Salvino da Silva, Marcela Rebecca Pereira, Robson José Silva Santana

Ferreira (2007) destaca que a crescente competitividade, os reflexos da abertura de mercado para os produtos estrangeiros e a necessidade de otimizar resultados demandam procedimentos de apropriação de custos indiretos que tornem os preços dos diversos produtos fabricados mais competitivos e explorem as tendências do mercado consumidor. Assim, apropriações inadequadas podem prejudicar sensivelmente o comportamento das vendas de seus produtos e até reduzir sua participação relativa no mercado.

\subsection{Gestão de Custos no Agronegócio no Frango}

Uma gestão eficiente de custos é imprescindível em qualquer atividade econômica. No agronegócio do frango, essa gestão precisa ser meticulosamente mais rigorosa, visto que a atividade está sujeita a inúmeros outros fatores, especialmente de ordem climática, que podem desestruturar todo um processo produtivo, chegando inclusive a descontinuar a produção.

Sobre uma boa gestão no agronegócio, em especial quanto à variabilidade dos custos, Raíces (2003) aponta que ser um bom produtor rural vai muito além de garantir qualidade, obter boa produtividade ou manter uma criação sadia. A atividade exige o conhecimento de todos os custos envolvidos na operação. O sucesso na agricultura, como em qualquer outra empresa, vem da atenção à gestão dos custos de produção, à capacitação do pessoal e à seleção de insumos.

A definição de eficiência da pecuária começa pela conjunção de despesas e receitas, gerando lucros ou prejuízo. Comumente, o produtor preocupa-se com o preço do produto, e o preço não justifica o sucesso ou fracasso de um negócio. O que interessa é o todo, custo em conjunto com preço e renda, o que determinará o lucro da atividade em questão (Carlini Junior \& Fonseca, 2006).

Segundo Arruda e Santos (2017), a produção brasileira de carne de frango totalizou, no ano de 2015, 13,146 milhões de toneladas de frango, volume 3,58\% superior ao registrado no ano de 2014. Com esse resultado, o Brasil se consolidou 
Análise da Relação entre Quantidades e Custos Totais de Produção de Frango no Estado do Ceará Jucimar Casimiro de Andrade, Fernando Salvino da Silva, Marcela Rebecca Pereira, Robson José Silva Santana

como segundo maior produtor de carne de frango do mundo, superando a China, que produziu, no ano de 2015, um total estimado de 13,025 milhões de toneladas.

A importância da análise de custos da pecuária também é abordada por Marion (2009), ao afirmar que conhecer o custo real de cada lote ou do rebanho a qualquer momento é uma informação imprescindível à gerência, não só para apurar a rentabilidade após a venda, mas também (o que é mais importante) para determinar o ponto ótimo de venda, ou seja, não manter o gado ou lote quando os custos passam a ser maiores que o ganho de peso (ou mantê-lo se o preço de mercado está baixo).

Mesmo com a crise econômica, a cadeia produtora e exportadora de carne de frango viveu um bom momento no ano 2015, em que o consumo per capita de carne atingiu índice médio de 43,25 quilos, saldo 1,1\% maior que o obtido no ano anterior de 2014 (Arruda \& Santos, 2017).

\begin{tabular}{|c|c|c|c|c|c|c|c|c|c|c|c|c|c|c|}
\hline Item do custo & Jan. & Fev. & Mar. & Abr. & Mai. & Jun. & Jul. & Ago. & Set. & Out. & Nov. & Dez. & Média & $\%$ \\
\hline Alimentação & 1,71 & 1,73 & 1,73 & 2,05 & 2,05 & 1,68 & 1,98 & 2,09 & 2,26 & 2,37 & 2,2 & 2,2 & 2,01 & 66,01 \\
\hline Mão de obra & 0,12 & 0,11 & 0,11 & 0,12 & 0,12 & 0,12 & 0,12 & 0,12 & 0,12 & 0,13 & 0,1 & 0,13 & 0,12 & 3,87 \\
\hline $\begin{array}{c}\text { Custo de } \\
\text { capital }\end{array}$ & 0,05 & 0,05 & 0,05 & 0,05 & 0,05 & 0,05 & 0,05 & 0,05 & 0,05 & 0,05 & 0,5 & 0,5 & 0,05 & 1,66 \\
\hline Depreciação & 0,05 & 0,05 & 0,05 & 0,05 & 0,05 & 0,05 & 0,05 & 0,05 & 0,05 & 0,05 & 0,5 & 0,5 & 0,05 & 1,69 \\
\hline Outros & 0,71 & 0,71 & 0,71 & 0,71 & 0,99 & 0,85 & 0,85 & 0,85 & 0,89 & 0,89 & 0,9 & 0,9 & 0,81 & 26,8 \\
\hline Total & 2,64 & 2,65 & 2,65 & 2,98 & 3,26 & 2,75 & 3,05 & 3,16 & 3,37 & 3,49 & 4,3 & 4,23 & 3,04 & 100 \\
\hline
\end{tabular}

Quadro 1. Composição do custo de produção - aviário climatizado positivo/Ceará Fonte: dados da pesquisa, 2017.

O Quadro acima refere-se à produção no estado do Ceará. Aponta que os maiores custos são decorrentes da alimentação das aves (66,01\%), seguidos de outros custos no valor de 26,8\%, e da mão de obra (3,87\%). Por não ser um estado produtor de um dos principais insumos usados da produção de frangos, o milho, os produtores muitas vezes têm de importar essa matéria-prima de outras localidades, e essa dependência logística faz com que o preço seja bastante suscetível às variações do mercado. 
Análise da Relação entre Quantidades e Custos Totais de Produção de Frango no Estado do Ceará Jucimar Casimiro de Andrade, Fernando Salvino da Silva, Marcela Rebecca Pereira, Robson José Silva Santana

Crepaldi (2009) destaca alguns desafios para a avicultura, principalmente em relação aos custos de produção:

Para ser competitivo, o avicultor precisa conhecer seus custos, ampliar a linha de produção e buscar parcerias. Na atualidade, é praticamente impossível o pequeno produtor sobreviver economicamente na avicultura sem unir-se às integrações, isto é, fazer parte do sistema de comercialização que garante a absorção do produto final, independentemente da situação do mercado.

No Nordeste, segundo estudos de Abramovay (2009), cerca de $60 \%$ do frango de corte produzido é proveniente do sistema de integração, em que os custos são compartilhados entre produtor e agroindústria. Mesmo assim, o produtor tem muita dificuldade, principalmente em relação ao acesso à assistência técnica e extensão rural.

A respeito da expansão da avicultura nordestina, Crepaldi (2009) destaca que existem ainda alguns problemas que impedem um maior crescimento do setor, sobretudo relacionados aos insumos, mas também que a atividade tem grandes perspectivas de mercado, tanto pela ampliação da demanda interna, quanto pela expansão do consumo mundial de proteína animal.

O autor aponta que as regiões Norte e Nordeste, devido aos pequenos volumes produzidos, provavelmente decorrentes de custos mais elevados de produção, em especial do custo de alimentação das aves, têm pouca participação na produção nacional, sendo que o Nordeste detém uma posição melhor do que a ocupada pela Região Norte.

\subsection{Evidências Empíricas Relacionadas à Gestão de Custos na Agropecuária}

Estudos anteriores mostram que o custo com alimentação responde por, aproximadamente, $70 \%$ do custo total de produção de frango de corte e $80 \%$ dos custos variáveis. Dessa forma, as decisões sobre alimentação afetam, em grande escala, a rentabilidade econômica da atividade (Oliveira, 2009). 
Análise da Relação entre Quantidades e Custos Totais de Produção de Frango no Estado do Ceará Jucimar Casimiro de Andrade, Fernando Salvino da Silva, Marcela Rebecca Pereira, Robson

José Silva Santana

Carmo, Santos e Santos (2008) realizaram um estudo com o objetivo de apresentar os resultados obtidos por meio da aplicação das metodologias de custeamento por absorção, custeamento variável e custeamento baseado em atividades $(A B C)$ em um empreendimento estrutiocultor (de criação de avestruz) da cidade de Uberaba - MG, cuja atividade operacional consistia na incubação, cria, recria e engorda de avestruzes para o abate e, ainda, comparar tais resultados para identificar aquela metodologia de custeamento que mostraria melhor adaptação à atividade pecuária em questão no processo decisório em geral. Constatou-se que a metodologia de custeamento baseado em atividades $(A B C)$ produziu a maior quantidade de informações relevantes para a tomada de decisões gerenciais nesse tipo de negócio pecuário.

Sell (2005) realizou um estudo com o objetivo de estabelecer uma função matemática que relacionasse os Custos Indiretos de Fabricação (CIF) com HorasMáquina (HM) e com Lotes de Produção (LP) de forma a mostrar como esses modelos matemáticos poderiam ser utilizados como instrumento de controle, auxiliando o administrador no processo de gestão empresarial. Foi apontado que o melhor modelo é o que utiliza as variáveis HM e LP como explicativas do CIF, e isso ficou demonstrado por meio dos testes realizados, a exemplo de $\mathrm{R}^{2}$ de $89,93 \%$.

Ainda no campo de pesquisa empírica aplicada à criação de aves, Hofer, Kipper e Silva (2006) elaboraram um estudo de caso sobre a atividade estrutiocultora, enfocando o controle de custos e a viabilidade econômica do negócio. Os autores aplicaram a metodologia de custeamento variável em uma propriedade na região oeste do Paraná, no período de 01 de julho de 2004 a 30 de junho de 2005. Concluiu-se que a atividade era viável para a empresa analisada; entretanto, necessitava da implantação de um sistema de controle de custos para auxiliar a tomada de decisões, bem como para controle do plantel.

Um estudo foi realizado por Corrêa, Benedicto, Carvalho, Ribeiro e Campos (2015) com o objetivo de analisar a elasticidade de transmissão de preços da carne de frango no mercado do estado de São Paulo e identificar em qual nível de mercado 
concentra-se o maior poder de formação de preços, seja ao nível do produtor, atacado e varejo. Para tanto, foi empregado no tratamento dos dados de preços da carne de frango ao nível do produtor, atacado e varejo, o modelo econométrico de Vetor AutoRegressivo (VAR). Os resultados encontrados por meio da análise da decomposição da variância dos erros de previsão demonstraram que o produtor possui alto poder de formação dos preços da carne de frango no mercado interno de São Paulo, tendo influência direta na formação dos preços praticados tanto em nível de atacado como de varejo.

Carmo et al. (2011) realizaram um estudo cujo objetivo era conceber uma modelagem matemática que fosse capaz de linearizar o comportamento dos custos totais de produção de frango em relação às quantidades produzidas de frango em cada lote no sistema de produção climatizado positivo no estado de Minas Gerais, como forma de constituir uma ferramenta de auxílio à tomada de decisão nessa atividade. $\mathrm{O}$ modelo por eles desenvolvido foi satisfatório para uma estimativa de produção compreendida entre 20.000 e 25.000 unidades/cabeças de frango desde que seja admitida uma margem de erro de $\pm 11 \%$.

Ainda nessa linha de investigação empírica, Melo, Andrade, Monteiro, Gudes e Silva (2015) realizaram um estudo semelhante ao de Carmo et al. (2011), cujo objetivo era analisar a relação estatística entre a quantidade produzida de cabeças de frango para abate e os custos totais de produção, que englobava os custos fixos e variáveis no estado de Pernambuco. Verificando que o modelo encontrado é satisfatório para uma estimativa de produção de frangos para abate entre 22.000 e 23.200 cabeças, desde que se assuma uma margem de erro de $\pm 3 \%$, aplicando o modelo em intervalos menores, percebeu-se ainda mais uma diminuição na margem de erro para os dados observados, sendo que o ponto ótimo de estimação encontra-se quando há uma produção mensal de em torno de 22.600 unidades.

Mas este estudo, igualmente ao anterior, foi limitado quanto aos meios, visto que utilizou uma série relativamente curta (2010 e 2014), o que, segundo os autores, pode ter ocasionado uma constante negativa que, teoricamente, teria que ser positiva, 
Análise da Relação entre Quantidades e Custos Totais de Produção de Frango no Estado do Ceará Jucimar Casimiro de Andrade, Fernando Salvino da Silva, Marcela Rebecca Pereira, Robson José Silva Santana

conforme se observa na literatura sobre custos e, igualmente ao trabalho anterior, também apresentou outras limitações que impossibilitaram maiores generalizações, por não realizar testes de especificação para validar o modelo encontrado.

\section{METODOLOGIA}

Em relação ao objetivo, esta pesquisa classifica-se como descritiva e explicativa e, quanto aos procedimentos, classifica-se em bibliográfica e documental. Conforme Prestes (2008), a pesquisa descritiva procura descrever as características de determinado fenômeno e a pesquisa explicativa visa identificar os fatores que contribuem para a ocorrência dos fenômenos. Em relação à pesquisa documental, ela é elaborada tomando-se como base materiais que ainda não receberam tratamento analítico.

Destarte, a Central de Informações de Aves e Suínos (CIAS) calcula e divulga mensalmente os custos de produção do produtor e da agroindústria pelo site da, nas modalidades: climatizado negativo, climatizado positivo e convencional. Para essa análise, utilizaram-se os dados secundários mensais de custos totais de produção (fixos e variáveis), tanto do produtor quanto das agroindústrias, no sistema de produção convencional (sistema mais adotado pelos produtores cearenses) entre os meses de janeiro de 2010 a setembro de 2014, no estado do Ceará. Esses dados foram coletados diretamente da CIAS, não havendo nenhum tipo de alteração ou transformação em seus valores, inclusive quanto a efeitos da inflação ou câmbio. Apenas se coletaram e interpretaram os dados.

Quanto ao método matemático que serviu de base para a análise, adotou-se o modelo econométrico Mínimos Quadrados Ordinários (MQO). Para Batalha (2010), a modelagem é uma arte e desenvolver um modelo que represente um sistema real é uma tarefa que requer muito cuidado e muita experiência. Um modelo deve ter duas qualidades: (1) ser descritivo, fornecendo explicações que facilitem a compreensão do 
sistema estudado; e (2) ser descritivo, representando um conselheiro que orienta sobre situações futuras.

A modelagem envolve duas situações de conflito que exigem que o modelo seja simples o suficiente para permitir sua construção e manipulação e, ao mesmo tempo, seja complexo o bastante para envolver todas as variáveis relevantes e suas relações. Uma forma de contornar esse conflito é construir modelos simples e sofisticá-los à medida que novas exigências forem surgindo (Corrar Paulo \& Dias Filho, 2009).

Assim, Carmo et al. (2011) efetuaram estimações por meio do MQO para os dados de custos de frango disponibilizados pela CIAS e conseguiram linearizar um modelo. Esse modelo foi replicado neste trabalho para os dados de custos de produção do estado do Ceará.

Utilizou-se, neste trabalho, o modelo dos Mínimos Quadrados Ordinários (MQO), sendo a variável dependente o custo total de produção de frangos e a variável independente a quantidade de frango produzida para abate em cada mês.

Para verificar a validade e confiabilidade dos dados, foram utilizados vários testes comumente aplicados na literatura como: Doornik-Hansen (1994), por meio do qual foi possível testar se a hipótese de normalidade era verdadeira; o teste de White (1980) e Breusch-Pagan (1979) para testar a presença de Heterocedasticidade dos dados e LMF (Multiplicador de Lagrange) e Teste Ljung-Box para a detectar a presença de autocorrelação residual.

Bruni (2010) destaca que o teste de White tem por objetivo verificar se o modelo apresenta erros ao longo de sua função, ou seja, se ocorre a perda do poder de explicação das variáveis e destaca que a Heterocedasticidade é um fenômeno em que, no modelo regredido, ocorre uma forte dispersão dos dados analisados em torno de uma reta; assim, na ocorrência de Heterocedasticidade, a variância dos erros e os coeficientes das variáveis explicativas tendem a não ser constantes.

A realização dos cálculos deu-se com o auxílio da planilha eletrônica Microsoft Excelße do Software Econométrico Gret/ßB - Gnu Regression, Econometrics and Timeseries Library, versão Gret/@-2016-64.exe. Para a análise estatística do grau de 
confiabilidade dos dados pela técnica dos Quadrados Mínimos Ordinários (MQO), utilizaram-se os coeficientes de correlação $R$ ( $R$ múltiplo) e determinação $R^{2}(R$ quadrado), além do teste "t de student" para testar a significância dos coeficientes angular e linear, respectivamente. Outrossim, foram utilizados os testes: $F$ de FischerSnedecor para verificar o grau de influência da variável explicativa sobre a explicada, teste de normalidade dos resíduos (Teste Doornik-Hansen, 1994), teste para verificar a presença de Heterocedasticidade (Teste de White e Breush-Pagan, 1980) e o teste Autocorrelação (Teste LMF e Ljung-Box), para verificar se os resíduos estão correlacionados e, consequentemente, enviesando os resultados encontrados (Corrar et al., 2009).

Para testar a hipótese de normalidade dos resíduos, foi utilizado o teste de Doornik-Hansen (2008), pois o mesmo apresenta propriedades estatísticas superiores ao de Jaque-Bera em pequenas amostras. Assim, esse teste assume as seguintes hipóteses:

$\mathrm{H}_{0}=$ os resíduos estão normalmente distribuídos; e

$\mathrm{H}_{1}=$ os resíduos não estão normalmente distribuídos.

Assim, a utilização de técnicas estatísticas para a construção de modelos que proporcionarão a projeção de custos tem como vantagens uma melhor capacidade de analisar o comportamento dos custos e o aumento da objetividade e confiabilidade na geração das informações contábeis (Sell, 2005).

Portanto, o uso de técnicas quantitativas e de softwares específicos dentro da contabilidade pode auxiliar a evidenciar melhor e solucionar problemas de forma mais rápida e eficaz. Então, considerando que os processos dentro de uma organização estão sempre se modificando, faz-se necessário o uso de técnicas e modelos estatísticos com poder de predição, principalmente como apoio à tomada de decisão empresarial sobre projeção de custos de produção, pois um dos objetivos principais da utilização de um modelo de ajuste linear, ou equação linear, é encontrar um modelo matemático que se ajuste à série de dados, com a utilização da memória 
Análise da Relação entre Quantidades e Custos Totais de Produção de Frango no Estado do Ceará Jucimar Casimiro de Andrade, Fernando Salvino da Silva, Marcela Rebecca Pereira, Robson

José Silva Santana

autorregressiva dos dados obtidos no passado, para então simular e projetar os possíveis cenários do futuro.

Uma observação a ser feita é que houve uma mudança na forma de divulgação dos custos de produção de frango pelo sistema CIAS. A partir de 2016, portanto, não se consegue mais visualizar os custos por unidade, por produtor e por agroindústria, o que explica a utilização da série de dados até setembro de 2015 para a análise desta pesquisa.

Após todas as estimações e testes estatísticos, foram feitas simulações no Microsoft Excel $\Theta$ com uso do modelo estimado, visando detectar o intervalo ótimo aproximado de cabeças a serem produzidas em cada plantel de frango. Para isso, utilizou-se o método de Simulação de Monte Carlo (MMC), que consiste em uma técnica matemática que possibilita levar em conta o risco envolvido em análises quantitativas, uma vez que fornece uma série de valores possíveis que auxiliam o tomador de decisão a analisar melhor o contexto dentro de um cenário incerto. Assim, as simulações apontam um gama de resultados possíveis dentro de parâmetros probabilísticos previamente estimados.

Destarte, apesar de o MQO ser o método mais utilizado na literatura para a resolução desse tipo de problema, o mesmo apresenta algumas limitações, especialmente em relação às probabilidades levantadas que podem não condizer com a realidade ou os parâmetros adotados que se mostraram fatores limitadores em função do tamanho reduzido da amostra. Outra limitação encontra-se no uso de apenas uma variável independente na estimativa do modelo (quantidade produzida).

\section{ANÁLISE E INTERPRETAÇÕES DE DADOS}

Devido à natureza quantitativa e multivariada dos dados de custos e quantidades de frango nas planilhas da CIAS, foram empregados procedimentos estatísticos multivariados de controle da média e da oscilação das variáveis observadas, com o objetivo de verificar se os parâmetros de distribuição seriam 
constantes em datas futuras, ou seja, se a oscilação dos valores de custos no passado teria um padrão mais homogêneo para futuras projeções na quantidade de lotes produzidos de frango. Desse modo, usou-se uma média anual das quantidades de cabeças produzidas de frango.

Para a obtenção das quantidades reais de frangos para abate utilizadas neste artigo, empregou-se o critério de Carmo et al. (2011) e Melo, Andrade, Monteiro, Gudes, \& Silva (2015), ou seja, uma regra de três simples que consistiu na divisão do custo variável total do produtor e da agroindústria pela média do custo variável unitário em cada ano. Por exemplo, no mês de setembro de 2015, a CIAS informou que os custos totais foram da ordem de $\mathrm{R} \$ 117.444,00$ e, para esse ano, a média dos custos variáveis (unidades produzidas de frango nos 12 meses de 2015) foi de $R \$ 7,69$ por cabeça. Procedeu-se aos cálculos aritméticos de $R \$ 117.444,00 / 7,69$ e obteve-se o valor aproximado de 15.278 cabeças por lote de $1.200 \mathrm{~m}^{2}$ como quantidades reais produzidas no mês de setembro de 2015. Logicamente, tal procedimento acaba por dissolver a série de dados, mas é o critério mais recomendado na literatura para equalizar os valores dos custos variáveis unitários e proporcionar melhores resultados com as estimações.

Esse procedimento foi feito para os meses de janeiro de 2010 a setembro de 2015, cujos dados encontram-se compilados na Tabela 1. 
Análise da Relação entre Quantidades e Custos Totais de Produção de Frango no Estado do Ceará Jucimar Casimiro de Andrade, Fernando Salvino da Silva, Marcela Rebecca Pereira, Robson José Silva Santana

Tabela 1

Custos totais e quantidades produzidas de frango de corte no Ceará ${ }^{1}$

\begin{tabular}{|c|c|c|c|c|c|c|c|c|c|c|c|c|}
\hline \multirow{2}{*}{ Mês } & \multicolumn{2}{|c|}{2010} & \multicolumn{2}{|c|}{2011} & \multicolumn{2}{|c|}{2012} & \multicolumn{2}{|c|}{2013} & \multicolumn{2}{|c|}{2014} & \multicolumn{2}{|c|}{2015} \\
\hline & $\mathbf{R} \$ /$ Lote & Cabeças & R\$/Lote & Cabeças & $\mathbf{R} \$ /$ Lote & Cabeças & R\$/Lote & Cabeças & R\$/Lote & Cabeças & $\mathbf{R} \$ /$ Lote & Cabeças \\
\hline 1 & $62.801,00$ & 13194 & $76.522,00$ & 19858 & $64.372,00$ & 15052 & $84.709,00$ & 12807 & $92.501,00$ & 13044 & $92.817,00$ & 12018 \\
\hline 2 & $62.836,00$ & 13197 & $73.963,00$ & 19166 & $70.119,00$ & 16444 & $84.709,00$ & 12807 & $93.486,00$ & 13186 & $93.383,00$ & 12092 \\
\hline 3 & $56.644,00$ & 11846 & $73.935,00$ & 19154 & $71.655,00$ & 16814 & $87.100,00$ & 13179 & $101.259,00$ & 14310 & $93.429,00$ & 12093 \\
\hline 4 & $56.672,00$ & 11849 & $72.806,00$ & 18849 & $71.182,00$ & 16693 & $83.705,00$ & 12651 & $102.946,00$ & 14554 & $104.765,00$ & 13601 \\
\hline 5 & $6.716,00$ & 11851 & $72.806,00$ & 18849 & $70.018,00$ & 16411 & $81.497,00$ & 12308 & $94.059,00$ & 13269 & $104.778,00$ & 13601 \\
\hline 6 & $58.645,00$ & 12269 & $72.174,00$ & 18680 & $70.175,00$ & 16449 & $84.120,00$ & 12716 & $98.337,00$ & 13844 & $101.729,00$ & 13193 \\
\hline 7 & $61.418,00$ & 12871 & $71.454,00$ & 18487 & $85.466,00$ & 20155 & $87.819,00$ & 13291 & $87.781,00$ & 12320 & $107.669,00$ & 13982 \\
\hline 8 & $63.926,00$ & 13412 & $74.312,00$ & 19289 & $89.926,00$ & 21236 & $91.366,00$ & 13842 & $87.240,00$ & 12241 & $111.524,00$ & 14495 \\
\hline 9 & $64.974,00$ & 13634 & $72.928,00$ & 18874 & $89.614,00$ & 21160 & $92.525,00$ & 14022 & $87.462,00$ & 12273 & $117.444,00$ & 15278 \\
\hline 10 & $69.684,00$ & 14654 & $74.466,00$ & 19283 & $91.406,00$ & 21608 & $91.305,00$ & 13832 & $92.417,00$ & 12988 & & \\
\hline 11 & $75.156,00$ & 15837 & $79.902,00$ & 20729 & $97.804,00$ & 23120 & $91.725,00$ & 13898 & $100.366,00$ & 14133 & & \\
\hline 12 & $75.166,00$ & 15837 & $80.014,00$ & 20729 & $97.941,00$ & 23120 & $99.505,00$ & 15107 & $101.504,00$ & 14270 & & \\
\hline
\end{tabular}

Nota. Fonte: dados da pesquisa, 2017.

Após tabulação com auxílio das planilhas do Microsoft Exce/ß, conforme Tabela 1, procedeu-se aos cálculos dos valores expressos dos anos de 2010 a 2015, em que a coluna produção (cabeças) representa a variável explicativa ( $\left.\boldsymbol{x}_{1}\right)$ quantidade produzida de frango para abate, e a coluna custo total ( $\mathrm{R} \$ /$ Lote) representa a variável resposta $(\boldsymbol{y})$ custo total de produção de frango para abate em cada lote de $1.200 \mathrm{~m}^{2}$ no estado de Ceará. O resumo da regressão desses valores feito com auxílio do Gret/ß está expresso na Tabela 2.

${ }^{1}$ Dados em milhares de reais (R\$). 
Análise da Relação entre Quantidades e Custos Totais de Produção de Frango no Estado do Ceará Jucimar Casimiro de Andrade, Fernando Salvino da Silva, Marcela Rebecca Pereira, Robson José Silva Santana

Tabela 2

Resumo dos resultados

\begin{tabular}{|c|c|}
\hline Tipo de Estatística & Valores \\
\hline R múltiplo & $-0,62$ \\
\hline R-Quadrado & 0,89 \\
\hline Estatística " $t$ " para testar a significância do intercepto (coeficiente linear) & $\begin{array}{c}5,899 \\
(1,38 \mathrm{e}-07) \\
* * *\end{array}$ \\
\hline Estatística " $t$ " para testar a significância do coeficiente angular & $\begin{array}{c}1,846 \\
(0,0694)^{*}\end{array}$ \\
\hline Estatística $F$ para testar o efeito da variável quantidade sobre a variável custo total & $\begin{array}{c}3,406873 \\
(-6,94 \mathrm{E}-02)\end{array}$ \\
\hline Durbin-Watson & 2,050084 \\
\hline $\begin{array}{l}\text { ** Significante a } 5 \% \\
{ }^{* * *} \text { Significante a } 1 \% \\
\text { Em parênteses as significâncias }\end{array}$ & \\
\hline
\end{tabular}

Nota. Fonte: dados da pesquisa, 2017.

Fica evidente que os custos totais de produção versus as unidades produzidas apresentam uma relação linear inversa. Assim, o modelo MQO apresentou um coeficiente de correlação ( $R$ múltiplo) em torno de -0,62, muito próximo de -1, indicando que existe um grau médio de relacionamento entre as variáveis custo total e quantidade produzida, ou seja, pode-se afirmar que o custo total de produção de frangos no estado do Ceará sofre uma variação em sentido oposto ao das quantidades produzidas. Assim, quanto mais se produz, existe uma tendência de diminuição dos custos totais (fixos e variáveis) para esse sistema de produção de frango para abate.

Passos (2010) aponta que tal resultado pode ser explicado pela economia de escala e, assim, há economia de escala quando o aumento do volume da produção de um bem por período reduz seus custos. Essa redução pode se dar pela possibilidade de utilização de métodos produtivos mais automatizados ou mais avançados, mas também pode estar relacionada a ganhos em propaganda, marketing, pesquisa e desenvolvimento (P\&D), financiamento, enfim em qualquer etapa da produção e comercialização. 
De acordo com Varian (2003), a economia de escala pode apresentar três tipos de retornos: o constante, o decrescente e o crescente.

- Constante: ocorre quando todas as unidades do fator variável que for aplicado ao fator fixo no sistema produtivo da empresa resultar em aumentos iguais no total de produção obtida;

- Decrescente: ocorre quando cada nova unidade do fator variável que for aplicada ao fato fixo aumentar menos a produção total que a unidade anterior;

- Crescente: ocorre quando cada unidade nova do fator variável que for aplicada ao fator fixo aumenta mais a produção total do que a unidade anterior.

Dessa forma, a economia de escala pode ser determinada como os ganhos que se verificam no produto e nos custos quando se aumenta a capacidade produtiva de uma empresa.

Quanto ao coeficiente de determinação (R-Quadrado) encontrado para o modelo, foi de 0,89 , o que significa que $89 \%$ do custo total podem ser explicados pela variável independente quantidade produzida $\left(\boldsymbol{x}_{1}\right)$. Os testes " $t$ " de significância dos parâmetros estimados, intercepto e coeficiente angular foram respectivamente de 5,9 e 1,85, um pouco elevados, mas com valor de $\mathrm{p}(1,38 \mathrm{e}-07$ e 0,0694$)$ menores que o nível de significância adotado nesta pesquisa, que foi de $\alpha=5 \%=0,05$. Destarte, rejeita-se a hipótese de efeito nulo da variável quantidade sobre a variável custo total. O teste $F$ Snedecor, que foi de aproximadamente 3,4, também rejeita a hipótese de não haver influência da variável quantidade sobre custo total ao nível de $5 \%$.

Os resultados dos cálculos efetuados pelo método MQO podem ser visualizados na tabela seguinte. 
Análise da Relação entre Quantidades e Custos Totais de Produção de Frango no Estado do Ceará Jucimar Casimiro de Andrade, Fernando Salvino da Silva, Marcela Rebecca Pereira, Robson José Silva Santana

Tabela 3

Coeficientes encontrados pelo modelo MQO

\begin{tabular}{lc}
\hline \multicolumn{1}{c}{ Coeficientes da equação } & Valores da equação \\
\hline Coeficiente Linear (constante $\left.\beta_{0}\right)$ & $95.217,60$ \\
Coeficiente Angular $\left(\beta_{1}\right)$ & $-0,48823$ \\
Equação pesquisada & $y=\beta_{0}+\beta_{1} x_{1}$ \\
\hline
\end{tabular}

Nota. Fonte: dados da pesquisa, 2017.

Após cálculo dos coeficientes e, encontrada a melhor equação que representa a relação entre as variáveis $\left(\boldsymbol{y} ; \boldsymbol{x}_{1}\right)$, procedeu-se aos testes econométricos de especificação para verificar se o modelo econométrico utilizado estava ajustado e respondia à variável de interesse $(y)$ por meio dos testes de normalidade dos resíduos, heterocedasticidade e de autocorrelação.

Para esse modelo, o valor do coeficiente linear $(95.217,60)$, que é representativo dos custos fixos dentro do modelo, apresentou um sinal positivo e o valor do coeficiente angular $(-0,48823)$, que representa a parcela variável dos custos totais, apresentou sinal negativo. Esse resultado corrobora o estudo de Sell (2005), ao apontar que o aumento de produção demonstrado pelas varáveis Horas-Máquinas (HM) e Lotes de Produção (LP) tende a manter os Custos Fixos de Fabricação com sinal positivo, ou seja, quanto mais se produz, maior será a tendência de estabilidade de custos fixos, mas com leve tendência de acréscimo.

Entretanto, os resultados mostraram-se divergentes aos achados de Carmo et al. (2011) e Melo et al. (2015), em que o intercepto apresentou sinal negativo e a tendência apresentou um sinal positivo em ambas as investigações. Carmo et al. (2011), ao realizar novas estimações com sinal positivo, observou que os resultados foram mais satisfatórios, com uma subestimação de apenas $+11 \%$. Para Melo et al. (2015), a manutenção dos sinais previamente encontrados para os custos fixos e variáveis mostrou-se mais satisfatória, com a diferença ficando em torno de $-13 \%$.

É interessante analisar as economias de escala de acordo com as razões que levam ao decréscimo dos custos unitários quando a produção é aumentada: os custos 
fixos são diluídos entre mais unidades; os custos e despesas de construção são reduzidos; os custos na compra de materiais e serviços podem ser cortados; e, além disso, melhorias e cortes de custos no processo produtivo podem ser obtidos (Schier, 2013).

Mendes e Padilha Junior (2007) acrescentam que a economia de escala é caracterizada quando os custos médios de uma empresa, no longo prazo, decrescem, à medida que o nível de produção e tamanho da empresa aumentam. Segundo eles, essa tendência está crescendo na agricultura brasileira, principalmente em relação à soja e ao algodão; e alertam que as economias de escala representam uma das principais barreiras à agricultura, pois impedem a entrada de novos concorrentes no mercado em virtude dos elevados custos, o que acaba por concentrar a produção em poucos e oligopolizar o mercado.

Assim, essas variações podem ser explicadas pela série utilizada pelos autores e pelo ganho de escala que cada estado tem para atender seus respectivos mercados consumidores de carne de frango.

Tabela 4

Testes de especificação para a série de dados

\begin{tabular}{lcccc}
\hline \multicolumn{1}{c}{ Testes } & Padrão & Resultado & Interpretação \\
\hline Normalidade & & Valor de $p>0,05$ & 0,4314 & Há normalidade \\
Heteroscedasticidade & Teste White & Valor de $p>0,05$ & 0,7077 & Há Homoscedasticidade \\
& Teste Breusch-Pagan & Valor de $p>0,05$ & 0,5831 & Há Homoscedasticidade \\
Autocorrelação & Teste LMF & Valor de $p>0,05$ & $6,78 \mathrm{E}-18$ & Há autocorrelação \\
& Teste Ljung-Box & Valor de $p>0,05$ & $3,79 \mathrm{E}-11$ & Há autocorrelação \\
\hline
\end{tabular}

Nota. Fonte: dados da pesquisa, 2017.

Esses testes de especificação foram calculados pelo pacote Gret/®, utilizando a série mencionada anteriormente, obtendo-se como resultado um Qui-quadrado (2) = 1,681 e um valor de $p=0,43143$; portanto, nesse caso, aceita-se a hipótese de normalidade dos resíduos. 
Para o cálculo do teste de Heterocedasticidade, foram utilizados os testes de White e Breush-Pagan, apesar de este ser recomendado apenas quando a série apresenta distribuição normal. Assim, esses testes tiveram a finalidade de identificar a não Homocedasticidade dos dados, ou seja, quando as variâncias não são iguais para todas as observações. Desse modo, o teste Breush-Pagan oferece um resultado baseado na estatística Qui-quadrado, nesse caso com valor de $\mathrm{p}=\mathrm{P}$ (Qui-quadrado(1) $>0,301237)=0,583108$, o que evidencia a hipótese nula de Homocedasticidade dos resíduos. Da mesma forma, o teste White com valor de $\mathrm{p}=\mathrm{P}$ (Qui-quadrado(2) > $0,452019)=0,797711$ também rejeita a hipótese de Heterocedasticidade.

Portanto, verificou-se, por meio dos testes acima, que o modelo estimado apresentou autocorrelação residual para as séries analisadas e, assim, utilizou-se a estimação de Cochrane-Orcutt com o objetivo de ajustar o modelo encontrado ao termo de erro dos resíduos, ou seja, foi feita correção da autocorrelação.

Após testes e encontrado o melhor modelo que se ajustou aos dados, procedeuse à estimação dos valores pelo modelo encontrado e procedeu-se à comparação com os valores reais dos custos e unidades do período de janeiro de 2014 a dezembro de 2014, conforme Tabela 5. 
Análise da Relação entre Quantidades e Custos Totais de Produção de Frango no Estado do Ceará Jucimar Casimiro de Andrade, Fernando Salvino da Silva, Marcela Rebecca Pereira, Robson José Silva Santana

Tabela 5

Verificação do modelo estimado com dados de 2014

\begin{tabular}{|c|c|c|c|c|}
\hline \multicolumn{5}{|c|}{ CUSTOS TOTAIS DE PRODUÇÃO (FIXO E VARIÁVEIS) } \\
\hline & \multicolumn{2}{|c|}{ VALORES REAIS } & \multicolumn{2}{|c|}{ ESTIMATIVA } \\
\hline Meses & $\begin{array}{l}\text { CUSTO TOTAL } \\
\text { (R\$/LOTE) }\end{array}$ & $\begin{array}{l}\text { PRODUÇÃO } \\
\text { EM CABEÇAS }\end{array}$ & $\begin{array}{l}\text { Valores Estimados } Y= \\
95.217,60+[-0,48823(X 1)]\end{array}$ & $\begin{array}{c}\text { Diferença [Estimado- } \\
\text { Real] }\end{array}$ \\
\hline jan/15 & $\mathrm{R} \$ 92.501,00$ & 13044 & $\mathrm{R} \$ 88.849,13$ & $-\mathrm{R} \$ 3.651,87$ \\
\hline $\mathrm{fev} / 15$ & $R \$ 93.486,00$ & 13186 & $\mathrm{R} \$ 88.779,80$ & $-R \$ 4.706,20$ \\
\hline $\operatorname{mar} / 15$ & $\mathrm{R} \$ 101.259,00$ & 14310 & $\mathrm{R} \$ 88.231,03$ & $-R \$ 13.027,97$ \\
\hline$a b r / 15$ & $R \$ 102.946,00$ & 14554 & $\mathrm{R} \$ 88.111,90$ & $-R \$ 14.834,10$ \\
\hline $\mathrm{mai} / 15$ & $\mathrm{R} \$ 94.059,00$ & 13269 & $\mathrm{R} \$ 88.739,28$ & $-R \$ 5.319,72$ \\
\hline jun/15 & $R \$ 98.337,00$ & 13844 & $R \$ 88.458,54$ & $-R \$ 9.878,46$ \\
\hline $\mathrm{jul} / 15$ & $\mathrm{R} \$ 87.781,00$ & 12320 & $\mathrm{R} \$ 89.202,61$ & $\mathrm{R} \$ 1.421,61$ \\
\hline ago/15 & $R \$ 87.240,00$ & 12241 & $R \$ 89.241,18$ & $R \$ 2.001,18$ \\
\hline set/15 & $\mathrm{R} \$ 87.462,00$ & 12273 & $R \$ 89.225,55$ & $R \$ 1.763,55$ \\
\hline out $/ 15$ & $R \$ 92.417,00$ & 12988 & $\mathrm{R} \$ 88.876,47$ & $-R \$ 3.540,53$ \\
\hline nov/15 & $R \$ 100.366,00$ & 14133 & $\mathrm{R} \$ 88.317,45$ & $-R \$ 12.048,55$ \\
\hline $\mathrm{dez} / 15$ & $R \$ 101.504,00$ & 14270 & $R \$ 88.250,56$ & $-R \$ 13.253,44$ \\
\hline Médias & $\mathrm{R} \$ 93.772,50$ & 13369 & & \\
\hline Totais & $\mathrm{R} \$ 1.139 .358,00$ & 160432 & $\mathrm{R} \$ 1.064 .283,48$ & $-\mathrm{R} \$ 75.074,52$ \\
\hline & \multicolumn{3}{|c|}{ Diferença entre valor Real e Estimado (\%) } & $-6,59$ \\
\hline
\end{tabular}

Nota. Fonte: dados da pesquisa, 2017.

Para essa comparação, utilizou-se a média dos custos totais de produção ( $R \$ 93.772,50$ ), o valor $R \$ 95.217,60$ (representando a parcela fixa dos custos) e o valor $\mathrm{R} \$-0,48823$ (representando a parcela variável dos custos), de forma a comparar a diferença gerada entre os custos totais incorridos em 2014 e os custos totais gerados pelo modelo ajustado. Assim, observa-se, em vermelho, uma diferença em torno de $6,59 \%$, uma subavaliação do modelo, que foi a menor diferença encontrada nas comparações. Caso fossem utilizados outros anos como parâmetro de comparação, os resultados não se mostrariam muito divergentes desse percentual.

Em seguida, foram feitas várias simulações com ao auxílio de planilhas eletrônicas, levando-se em consideração a quantidade média produzida entre os anos de janeiro de 2010 a setembro de 2015 para comparação com os valores reais do ano de 2014, pelo modelo $\boldsymbol{y}=95.217,60+\left(-0,48823 x_{1}\right)$, cujo gráfico expõe os resultados: 


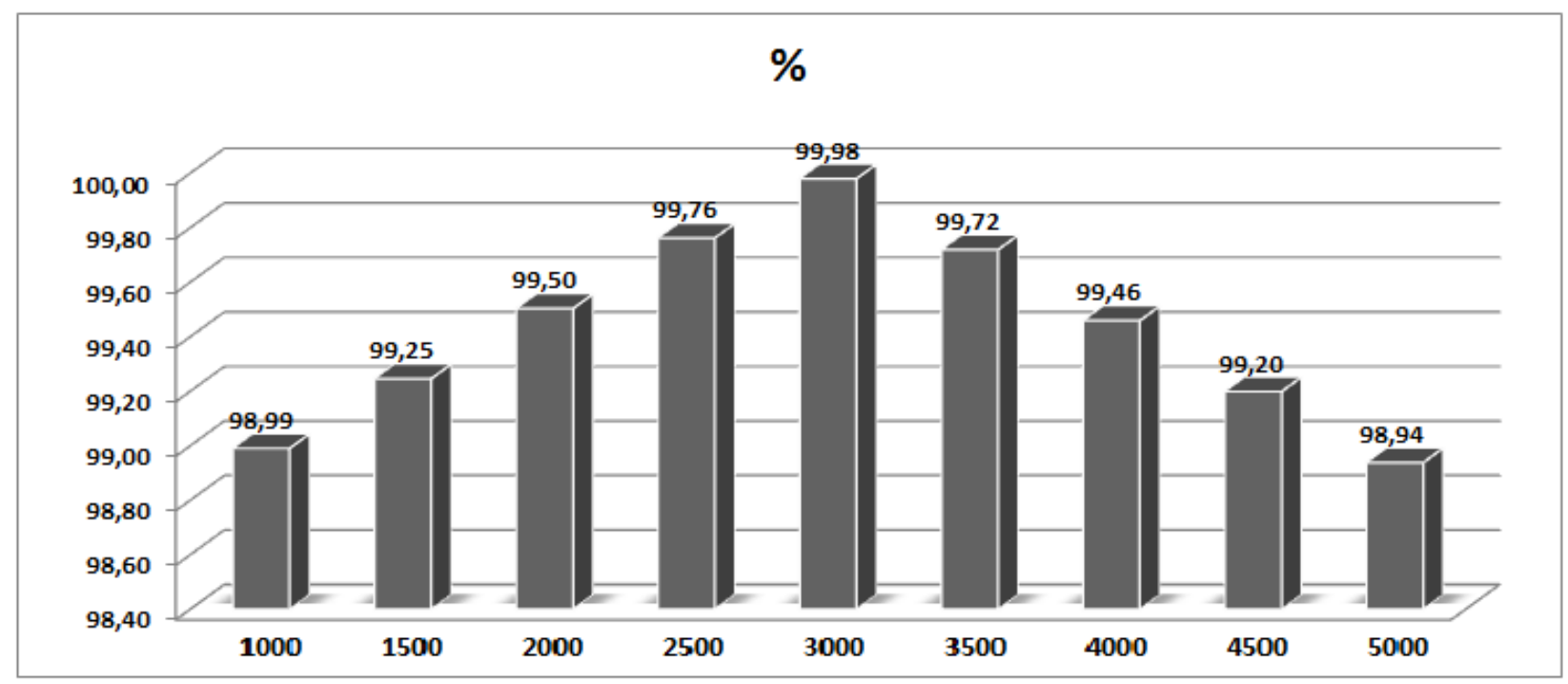

Gráfico 1. Grau de precisão do modelo encontrado (\%)

Fonte: dados da pesquisa, 2017.

Como discutido na metodologia, as simulações para encontrar os níveis aproximados de valores para a variável independente (quantidade a se produzir de frangos) foram feitas por meio da Simulação de Monte Carlo (MMC) e, além disso, o próprio Microsoft Exce/ß disponibiliza funções matemáticas que realizam essas estimações. Assim, as estatísticas utilizadas na determinação desse gráfico levam em consideração não apenas o valor atual das variáveis observadas, mas também seus valores anteriores, o que aumentou a eficácia do processo de monitoramento da variabilidade ao longo do tempo. Então, foi possível constatar que o modelo é quase preciso (em torno de 99,98\%) quando a produção atinge uma quantidade média de frangos produzidos de 3.000 aves por lote. De posse dessa informação, notou-se que, efetuando mais simulações com intervalos de \pm 500 unidades, o modelo tem uma oscilação no erro, em média, de aproximadamente $\pm 1,10 \%$. 


\section{CONSIDERAÇÕES FINAIS}

Os resultados obtidos pelas estimações apontaram para um modelo capaz de estimar custos de produção de frango (fixos e variáveis) no sistema de produção convencional no estado do Ceará. Esse modelo pode servir de suporte à tomada de decisão tanto pelo produtor quanto pela agroindústria, desde que se assuma produção em lotes de $1200 \mathrm{~m}^{2}$ entre 1.000 a 5.000 cabeças de frango, em que o erro de estimação ficou em torno de $-6,59 \%$.

Ao final, as simulações em planilhas apontaram que o modelo é quase preciso para uma produção em lotes de 3.000 aves. Esses resultados são importantes, pois permitem ao produtor e à agroindústria uma estimação de custos totais (fixos e variáveis) para uma dada quantidade de produção de frango, verificando, assim, se é vantajoso produzir ou não de acordo com as condições de preços praticadas no mercado local, ou seja, do estado do Ceará e região.

No entanto, apesar dos bons resultados obtidos por meio das estimações, devese ter cautela e evitar maiores generalizações, uma vez que a literatura ainda carece de mais evidências empíricas sobre o real comportamento dos custos produtivos nessa atividade pecuária, principalmente quanto ao comportamento dos custos fixos que, em decorrência de características peculiares adotadas no sistema de integração, parecem se comportar de maneira distinta em diferentes estados do país.

Outra limitação aos resultados repousa na pouca quantidade de variáveis utilizada como preditoras dos custos totais e na pouca quantidade de estudos similares à problemática aqui relatada. Assim, somente mais pesquisas empíricas podem suportar essas premissas, validando ou invalidando as investigações até agora realizadas.

Desse modo, espera-se que o modelo desenvolvido sirva de suporte para a melhoria nos mecanismos de planejamento de produção e controle de custos por parte dos integradores e integrados que produzem e processam frango no estado do Ceará, além de auxiliar a entender melhor os problemas fundamentais de projeção de custos, 
Análise da Relação entre Quantidades e Custos Totais de Produção de Frango no Estado do Ceará Jucimar Casimiro de Andrade, Fernando Salvino da Silva, Marcela Rebecca Pereira, Robson José Silva Santana

evitando desperdício de insumos, tempo e energia e eliminando, assim, aqueles recursos ineficazes e desnecessários à atividade produtiva.

Finalmente, tomando-se por base as informações obtidas e tratadas, provenientes dos órgãos oficiais do governo, espera-se que seja possível promover futuramente estudos mais homogêneos, levando-se em consideração outros itens não constantes e não tratados nesta pesquisa, mas que podem exercer influência na formação do custo total de produção do frango e, consequentemente, interferir no preço final de venda, como as variáveis: taxa de juros, taxa de câmbio, custos dos insumos usados na ração ou, até mesmo, preços de insumos praticados em outros estados do país.

\section{REFERÊNCIAS}

Arruda, L. L., \& Santos, C. J. (2017). Contabilidade Rural. (1a ed.). Curitiba: Intersaberes.

Batalha, M. O. (2010). Gestão agroindustrial. (3a ed.). São Paulo, Atlas.

Bruni, A. L. (2010). Estatística aplicada à gestão empresarial. (2a ed.). São Paulo, Atlas.

Carlini Junior., R. J., \& Fonseca, A. B. (2006) Custos como determinante para a competitividade do setor avícola: um estudo no município de São Bento do Uma PE. Revista custos e agronegócio online. v(2), n(1), 16-28.

Carmo, C. R. S., Lima I. G., Oliveira, R., \& Oliveira, L. F. M. (2011). Modelagem matemática na gestão de custos: um estudo acerca do comportamento dos custos na produção de frangos em Minas Gerais. Revista custos e agronegócio online. $v(7), n(2), 120-142$.

Carmo, M. S., Santos, C. M., \& Santos, L. M. S. (2008). Custos na estrutiocultura: uma comparação entre metodologias tradicionais de custeio e o ABC. Congresso Brasileiro de Custos, Curitiba, PR, Brasil, 15.

Central de Inteligência de Aves e Suínos (Cias). Recuperado de: <http://www.cnpsa.embrapa.br/cias/>. Acesso em: 05/dez/2017. 
Análise da Relação entre Quantidades e Custos Totais de Produção de Frango no Estado do Ceará Jucimar Casimiro de Andrade, Fernando Salvino da Silva, Marcela Rebecca Pereira, Robson

José Silva Santana

Corrar, L. J., Paulo, E., \& Dias Filho, J. M. D. (coordenadores). (2009). Análise multivariada para os cursos de administração, ciências contábeis e economia. (1a ed.). São Paulo, Atlas.

Corrêa, U., Benedicto, G. C., Carvalho, F. de M., Ribeiro, B. P. V. B., \& Campos, R. S. (2015). Estudo dos custos e receitas de laboratórios de produção e prática do Instituto Federal de Ciência e Tecnologia de Minas Gerais, Campus Bambuí. Congresso Brasileiro de Administração, Economia e Sociologia Rural (SOBER), João Pessoa, PB, Brasil, 53.

Crepaldi, S. A. (2009). Contabilidade rural - uma abordagem decisional. (5a ed.). São Paulo, Atlas.

Ferreira, J. A. S. (2007). Contabilidade de custos. São Paulo, Pearson Prentice Hall.

Ferreira, R. J. (2009). Contabilidade avançada e intermediária. (3a ed.). Rio de Janeiro, Ferreira.

Garcia, L. A. F. (2005). Economias de escala na produção de frangos no Brasil. Revista Economia e Sociologia Rural (RESR), v(4)3, n(3), 466-483.

Gomes, I. S., De Lima, D. H. S., \& Steppan, A. I. B. (2007, dezembro). Análise do Comportamento dos Custos Hospitalares Indiretos: Uma Investigação Empírica do Custo Hospitalar de Energia Elétrica no Setor de Radioterapia da Liga Norte-RioGrandense Contra o Câncer. Congresso Brasileiro de Custos ( $A B C)$, João Pessoa, PB, Brasil, 14.

Hofer, M. S., Kipper, C. M., \& Silva, L. M. S. (2006, outubro). Custos de produção aplicados à atividade da estrutiocultura. Congresso Brasileiro de Custos (CBC), Belo Horizonte, MG, Brasil, 13.

Marion, J. C. (2009). Contabilidade rural. (10a ed.). São Paulo, Atlas.

Martins, E. (2003). Contabilidade de custos. (9a ed.) São Paulo, Atlas.

Melo, A. de S., Andrade, J. C. de., Monteiro, D. S., Gudes, R. E. F. F., \& Silva, R. M. da. (2015, julho). Análise de custos na Gestão Rural: um estudo acerca do comportamento dos Custos de produção de frango em Pernambuco. Congresso Brasileiro de Administração, Economia e Sociologia Rural (SOBER), João Pessoa, PB, Brasil, 53.

Mendes, J. T. G., \& Padilha Junior, J. B. (2007). Agronegócio - uma abordagem econômica. São Paulo, Pearson Prentice Hall. 
Nogueira, M. P. (2004). Gestão de custos e avaliação de resultados: agricultura e pecuária. Bebedouro, Scot Consultoria.

Oliveira, N. C. de. (2009). Contabilidade do agronegócio. (1a ed.). Curitiba, Juruá.

Passos, M. S. (2010). Concorrência e competitividade: notas sobre estratégia e a dinâmica seletiva na economia capitalista. (Tese de Doutorado). Universidade Estadual de Campinas - UNICAMP, Campinas, SP, Brasil.

Prestes, M. L. de M. (2008). A pesquisa e a construção do conhecimento - do planejamento aos textos, da escola à academia. (3a ed.). São Paulo, Rêstel.

Raíces, C. (2003). Guia valor econômico de agronegócios. (1a ed.). São Paulo, Globo.

Reis, R. P. (2002). Fundamentos de economia aplicada. Lavras, UFLA/FAEPE.

Santos, J. dos., Marion, J. C., \& Segatti, S. (2009). Administração de custos na agropecuária. (4a ed.). São Paulo, Atlas.

Sell, I. (2005). Utilização da regressão linear como ferramenta de decisão na gestão de custos. Congresso Brasileiro de Custos (CBC), Florianópolis, SC, MG, Brasil, 9.

Data de Submissão: 16/07/2018

Data de Aceite: 03/04/2019 